\title{
Ten weeks of high-intensity interval walk training is associated with reduced disease activity and improved innate immune function in older adults with rheumatoid arthritis: a pilot study
}

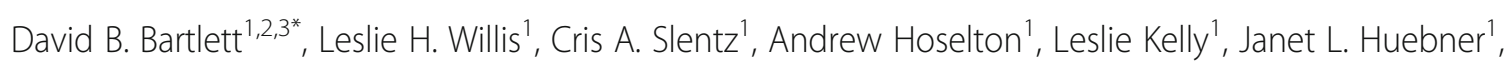
Virginia B. Kraus ${ }^{1}$, Jennifer Moss ${ }^{1}$, Michael J. Muehlbauer', Guillaume Spielmann ${ }^{4}$, William E. Kraus', Janet M. Lord ${ }^{3,5}$ and Kim M. Huffman ${ }^{1}$

\begin{abstract}
Background: Rheumatoid arthritis (RA) is a chronic inflammatory disease in which adults have significant joint issues leading to poor health. Poor health is compounded by many factors, including exercise avoidance and increased risk of opportunistic infection. Exercise training can improve the health of patients with RA and potentially improve immune function; however, information on the effects of high-intensity interval training (HIIT) in RA is limited. We sought to determine whether 10 weeks of a walking-based HIIT program would be associated with health improvements as measured by disease activity and aerobic fitness. Further, we assessed whether HIIT was associated with improved immune function, specifically antimicrobial/bacterial functions of neutrophils and monocytes.

Methods: Twelve physically inactive adults aged $64 \pm 7$ years with either seropositive or radiographically proven (bone erosions) RA completed 10 weeks of high-intensity interval walking. Training consisted of $3 \times 30$-minute sessions/week of ten $\geq 60$-second intervals of high intensity $\left(80-90 \% \mathrm{VO}_{2 \text { reserve }}\right)$ separated by similar bouts of lower-intensity intervals $\left(50-60 \% \mathrm{VO}_{2}\right.$ reserve). Pre- and postintervention assessments included aerobic and physical function; disease activity as measured by Disease Activity score in 28 joints (DAS28), self-perceived health, C-reactive protein (CRP), and erythrocyte sedimentation rate (ESR); plasma interleukin (IL)-1 $\beta$, IL-6, chemokine (C-X-C motif) ligand (CXCL)-8, IL-10, and tumor necrosis factor (TNF)-a concentrations; and neutrophil and monocyte phenotypes and functions.

* Correspondence: david.bartlett@duke.edu

'Duke Molecular Physiology Institute, Duke University School of Medicine,

Durham, NC, USA

2Division of Medical Oncology, Duke University School of Medicine, Durham,

NC 27701, USA

Full list of author information is available at the end of the article

(c) The Author(s). 2018 Open Access This article is distributed under the terms of the Creative Commons Attribution 4.0 International License (http://creativecommons.org/licenses/by/4.0/), which permits unrestricted use, distribution, and reproduction in any medium, provided you give appropriate credit to the original author(s) and the source, provide a link to the Creative Commons license, and indicate if changes were made. The Creative Commons Public Domain Dedication waiver (http://creativecommons.org/publicdomain/zero/1.0/) applies to the data made available in this article, unless otherwise stated. 
(Continued from previous page)

Results: Despite minimal body composition change, cardiorespiratory fitness increased by $9 \%$ (change in both relative and absolute aerobic capacity; $p<0.001$ ), and resting blood pressure and heart rate were both reduced (both $p<0.05$ ). Postintervention disease activity was reduced by $38 \%$ (DAS28; $p=0.001$ ) with significant reductions in ESR and swollen joints as well as improved self-perceived health. Neutrophil migration toward CXCL-8 ( $p=0.003)$, phagocytosis of Escherichia coli $(p=0.03)$, and ROS production $(p<0.001)$ all increased following training. The frequency of cluster of differentiation 14-positive $\left(C D 14^{+}\right) / C D 16^{+}$monocytes was reduced $(p=0.002)$, with both nonclassical (CD14 ${ }^{\text {dim }} /$ CD16 ${ }^{\text {bright }}$ ) and intermediate (CD14 ${ }^{\text {bright }} /$ CD16 ${ }^{\text {positive }}$ ) monocytes being reduced (both $\left.p<0.05\right)$. Following training, the cell surface expression of intermediate monocyte Toll-like receptor 2 (TLR2), TLR4, and HLA-DR was reduced (all $p<0.05$ ), and monocyte phagocytosis of $E$. coli increased $(p=0.02)$. No changes were observed for inflammatory markers IL-1 $\beta, I L-6, C X C L-8, I L-10, C R P$, or TNF-a.

Conclusions: We report for the first time, to our knowledge, that a high-intensity interval walking protocol in older adults with stable RA is associated with reduced disease activity, improved cardiovascular fitness, and improved innate immune functions, indicative of reduced infection risk and inflammatory potential. Importantly, the exercise program was well tolerated by these patients.

Trial registration: ClinicalTrials.gov, NCT02528344. Registered on 19 August 2015.

Keywords: Rheumatoid arthritis, Disease activity, Innate immunity, Inflammation, High-intensity interval exercise

\section{Background}

Rheumatoid arthritis (RA) is a chronic inflammatory disease characterized by swollen and painful joints, synovial inflammation, deformation of cartilage and bone structures, and a dysfunctional autoreactive immune system [1]. Highly differentiated, apoptosis-resistant innate immune cells characterize the pathology of RA; neutrophils dominate synovial fluid, and monocyte/macrophages infiltrate the expanded synovium. The proinflammatory milieu augments disease pathology by extending the lifespan of these cells and ensuring their retention in the joint. In the peripheral blood, immune cells show evidence of accelerated immune aging, with patients displaying a level of cell senescence/exhaustion suggestive of an adult several years older [2].

As such, in patients with RA, dysfunctional peripheral blood neutrophil migration promotes both nonspecific tissue damage and poor resolution of infection [3, 4]. Additionally, neutrophil phagocytic capacity is reduced, leading to increased risk of infections [5]. Although neutrophils in the joint produce more ROS [6], in the peripheral blood neutrophil ROS are similar or reduced compared with those of healthy control subjects [5, 7]. Furthermore, as compared with healthy individuals, peripheral blood monocyte/macrophages in patients with RA are characterized by an increased frequency of proinflammatory monocytes (cluster of differentiation 14-positive $\left.\left[\mathrm{CD} 14^{+}\right] / \mathrm{CD} 16^{+}\right)$in the circulation [8-11]. These cells play a major pathogenic role in RA, and reduction of their frequency may be of benefit to patients with RA [12, 13]. Further, blood monocytes have reduced phagocytic and antigen presentation capacity in $\mathrm{RA}$, adding to the risk of infection associated with poor neutrophil function [14]. As such, RA is associated with an impaired peripheral immune system compounded by immunosuppressive medications, leading to an increased risk of opportunistic infections, both bacterial and viral [15]. Therefore, identifying ways to reduce these risks and improve antibacterial immunity is critical to improving the health and quality of life of adults with RA.

In addition to a dysfunctional immune system, people with RA are often severely physically inactive owing to issues with pain and fatigue; inactivity leads to poor muscle function, low cardiorespiratory fitness, and worsened disability [16]. Current U.S. health guidelines and the American College of Rheumatology both suggest resistance exercises to improve muscle strength and quality and aerobic exercises to improve cardiorespiratory fitness. Although it is unclear whether any form of exercise can improve disease activity scores, there is a general consensus that short- and long-duration aerobic and resistance exercise programs do not worsen them [17].

In persons without RA, one of the underlying mechanisms by which exercise promotes improved health outcomes is through its anti-inflammatory effects $[18,19]$. Anti-inflammatory effects are promoted by a range of intrinsically related factors. Alongside improved metabolic features associated with muscle and adipose tissue, improvements in peripheral primary immune function have the potential to limit inflammatory insult [18, 20-23]. Although it is unclear which factor has the largest impact, each has individual health benefits. We have recently shown that in older healthy adults, better directional neutrophil migration toward the chemokine ( $\mathrm{C}-\mathrm{X}-\mathrm{C}$ motif) ligand (CXCL-8) is associated with increased physical activity, suggesting that exercise training might improve neutrophil migratory functions [20]. Blood monocyte phenotype can be modified by aerobic and resistance exercise training, 
suggesting that exercise training may improve the dysregulated monocyte/macrophage inflammatory potential in patients with RA [24-26]. Therefore, exercise programs that improve cardiovascular fitness and muscle function may improve long-term health in patients with RA by modifying immune function.

High-intensity interval training (HIIT) is an exercise activity that may improve the health of patients with RA. In persons without RA, HIIT is equivalent or superior to moderate-intensity continuous training at improving cardiorespiratory fitness when matched for energy expenditure or time spent in the activity $[25,27]$. HIIT also improves immune cell functions, specifically peripheral blood neutrophil and monocyte functions $[25,28,29]$. Critically, HIIT does not increase concentrations of systemic inflammatory cytokines, which may be detrimental to patients with RA [25]. However, few studies have assessed HIIT in RA to modify disease activity, cardiorespiratory fitness, physical function, or immune activity. Those which have suggest that HIIT is both safe and well tolerated and can rapidly increase cardiorespiratory fitness and muscle strength and also improve joint health [30-32].

Therefore, the purpose of this pilot study was to determine the efficacy of 10 weeks of HIIT walking for improving disease activity and aerobic capacity in older (> 55 years) adults with low to moderate RA. Additionally, we aimed to assess the effects of HIIT walking on peripheral blood neutrophil and monocyte antibacterial function and systemic inflammatory cytokine concentrations, as well as to generate effect size estimates for a larger clinical trial. We hypothesized that HIIT would increase aerobic capacity and reduce disease activity.

\section{Methods}

\section{Participants}

Twelve sedentary participants (11 women and 1 man) aged $64 \pm 7$ years and with confirmed and stable RA were recruited for the study. Participants were either seropositive or had radiographic joint erosions of finger joints, met the 1987 American College of Rheumatology criteria for RA [33], had no medication changes in the previous 3 months, and were using prednisone $\leq 5 \mathrm{mg} / \mathrm{d}$. Exclusions were known diabetes mellitus or cardiovascular disease and an inability to walk unaided on a treadmill. All participants gave written informed consent, and the study was approved by the Duke University Medical Center Institutional Review Board (IRB no. Pro00064057).

\section{Exercise training}

Exercise training consisted of 10 weeks of $3 \times 30$-minute sessions per week of supervised treadmill walking. In aggregate, participants completed 99\% of prescribed exercise sessions. Exercise intensities were determined from a cardiorespiratory fitness test. For exercise prescription, $\mathrm{VO}_{2}$ reserve was chosen and calculated as previously described [34]. Participants were given between three and six sessions to become accustomed to the exercise (30- to 45-second intervals at target heart rates; total time, 20 minutes). Exercise consisted of a 5-minute warm-up and 5-minute cool-down as part of the total session. Intervals were designed to elicit a heart rate corresponding to $80-90 \%$ of $\mathrm{VO}_{2}$ reserve (high intensity = actual heart rate percentage of $85 \pm 5 \%$ ) and $50-60 \%$ $\mathrm{VO}_{2}$ reserve (active recovery). Speeds did not exceed walking pace (range, 1-4.6 $\mathrm{mph}$ ), and if heart rate was not achieved by walking speed, gradient (range, 2-15\%) was added to increase heart rate. High-intensity intervals were between 60 and 90 seconds, followed by active recovery intervals of a similar duration. Rather than controlling each session for energy expenditure, total intervals per session were adjusted so that the exercise duration per session was 30 minutes. Ratings of perceived exertion were detailed at the end of each high-intensity interval bout.

\section{Assessment of disease activity}

All participants were examined by a rheumatologist using previously described methods for determining disease activity using the Disease Activity Score in 28 joints (DAS28) [35]. This score includes assessment of the number of swollen and tender joints, a visual analogue scale for general health, and C-reactive protein (CRP) concentration or erythrocyte sedimentation rate (ESR) (DAS28 $8_{\mathrm{CRP}}$ and DAS28 $8_{\mathrm{ESR}}$, respectively).

\section{Fitness, function, and body composition}

Exercise treadmill testing was used to assess cardiorespiratory fitness. Aerobic capacity $\left(\mathrm{VO}_{2 \text { peak }}\right)$ was determined by a graded maximal treadmill test starting at 2 $\mathrm{mph} / 0 \%$ grade and then increasing speed and/or grade such that the metabolic demand increased at approximately $3.5 \mathrm{ml} / \mathrm{kg} / \mathrm{min}$ until volitional exhaustion. Twelve-lead electrocardiogram, ventilation, and gas exchange were continuously assessed and recorded as 15-second averages using a Parvo Metabolic Cart (Parvo Medics, Sandy, UT, USA). Highest 15-second values were used for determination of $\mathrm{VO}_{2 \text { peak }}$, and a valid test was confirmed by either a respiratory exchange ratio (RER) $>1.1$ (mean RER, $1.2 \pm 0.1$ at both times) or a rating of perceived exertion $\geq 17$. Body composition was assessed according to Siri's three-compartmental model [36]. Body weight, fat mass, and lean mass were determined by air displacement plethysmography (BOD POD System; COSMED, Rome, Italy) [37]. Resting blood pressure and heart rate were taken following 15 minutes of sitting quietly. Each participant also completed a short battery of physical functioning tests that are indicative of health and frailty in older adults [38-40]. Grip strength 
was assessed by dynamometry in both hands in triplicate; the best score was taken. Timed Up and Go (TUG), Berg Balance Scale, 30-second chair stands, and 400-m walk tests were all supervised by a trained exercise physiologist. All tests were completed before training and at least 24 hours after the last exercise session.

\section{Physical activity and health questionnaires}

During their fasting blood draw visits (see below) at baseline and following the intervention, participants completed standardized validated physical activity and health questionnaires. Physical activity questionnaires were used to determine that participants were not engaging in more physical activity than what was prescribed in the exercise program. Questionnaires used included the Incidental and Planned Exercise Questionnaire (IPEQ), the Stanford Brief Activity Survey (SBAS), the Hospital Anxiety and Depression Scale (HADS), a visual analogue scale for pain (VAS), and the Health Assessment Questionnaire Disability Index (HAQ-DI).

\section{Fasting glucose and insulin}

Blood was obtained following a 10-hour overnight fast both before training and between 16 and 24 hours after the last exercise bout. Glucose was measured with a YSI Biochemistry Analyzer (YSI Inc., Yellow Springs, OH, USA), and insulin was measured by enzyme-linked immunosorbent assay (ELISA) according to the manufacturer's guidelines (Thermo Fisher Scientific, Waltham, MA, USA); both were measured in duplicate. Hemoglobin A1C (HbA1c) values were obtained through a commercial clinical analysis company (LabCorp, Burlington, NC, USA). The homeostasis model assessment of insulin resistance (HOMA-IR) was calculated as described previously using the HOMA2-IR online calculator [41, 42].

\section{Immune cell isolation}

Complete blood count differentials were measured using a commercial clinical analysis company (LabCorp). Neutrophils were isolated from heparin-treated blood following $2 \%$ dextran sedimentation and separation on a discontinuous Percoll gradient as previously described [20]. Neutrophil purity and viability were determined by Giemsa staining (Diff-Quik; Gentaur Europe, Kampenhout, Belgium) and trypan blue exclusion, respectively. Neutrophil purity and viability were consistently $\geq 95 \%$. Neutrophils were resuspended at $3 \times 10^{6} \mathrm{cells} / \mathrm{ml}$ in either RPMI 1640 (+calcium, +magnesium: RPMI) medium only (Sigma-Aldrich, St. Louis, MO, USA), RPMI 1640 containing $0.15 \%$ bovine serum albumin (BSA; RPMI-BSA; Sigma-Aldrich) or $\mathrm{HBSS}+/+$ (+ calcium, + magnesium) medium, depending on the assay.

Peripheral blood mononuclear cells (PBMCs) were isolated by Ficoll-Paque (GE Healthcare Life Sciences,
Marlborough, MA, USA) density gradient centrifugation. Briefly, blood was diluted 1:1 with RPMI 1640 and layered over the Ficoll before centrifugation at $400 \times g$ for 30 minutes at room temperature. The mononuclear cell layer was removed and washed twice before being resuspended at $1 \times 10^{6}$ cells $/ \mathrm{ml}$ in RPMI $+1 \%$ BSA.

\section{Neutrophil migration}

Neutrophil migratory dynamics were assessed using an Insall chamber (Weber Scientific International Ltd., Teddington, UK) as previously described $[4,43]$. Briefly, coverslips were coated with $7.5 \%$ culture-tested BSA (Sigma-Aldrich), and neutrophils were suspended in RMPI-BSA adhered to this surface for 20 minutes at room temperature. The use of this coating of BSA has previously been shown to mimic the ligand for CD11b and CD18, intercellular adhesion molecule (ICAM)-1 [44]. The coverslip was then inverted on the Insall chamber before addition of buffer (RPMI) alone as a control or buffer containing $100 \mathrm{nM}$ CXCL-8 (R\&D Systems, Minneapolis, MN, USA).

Neutrophil migration was monitored using a Zeiss Axiovert 100 inverted microscope (Carl Zeiss Microscopy, Buffalo Grove, IL, USA) fitted with a Hamamatsu ORCA 100 digital camera (Hamamatsu, Japan). Time-lapse recordings and calculations of neutrophil migratory dynamics were performed as previously described [4]. Briefly, the Insall chamber allows the formation of stable chemoattractant gradients, with defined, consistent direction in the $y$ direction for each experiment [43]. Only distance traveled in the $y$ direction over time was included in calculations of chemotaxis. Migration was assessed using three parameters: average cell speed $(\mu \mathrm{m} / \mathrm{min})$ of movement toward the chemokine (termed chemokinesis), average velocity $(\mu \mathrm{m} / \mathrm{min})$ of cells (termed chemotaxis), and accuracy of movement (termed chemotactic index). Chemotactic index is expressed in a comparative scale and arbitrary units (a.u.) ranging from -1 to +1 . Movement directly toward the chemoattractant is +1 , whereas movement directly away is -1 .

Recordings lasted 20 minutes per experiment, with 20 slides captured using OpenLab software (Improvision, Coventry, UK). The Java software ImageJ (Wayne Rasband, National Institutes of Health, Bethesda, MD, USA) was used to analyze cell tracks. All analyses were carried out by a single analyst blinded to subject group and cell conditions.

\section{Bacterial phagocytosis}

Phagocytosis of opsonized fluorescein isothiocyanate (FITC)-labeled Escherichia coli (Thermo Fisher Scientific) was assessed in whole blood as previously described [20]. Briefly, phagocytosis was assessed in heparin-treated whole blood incubated for 10 minutes at $4{ }^{\circ} \mathrm{C}$ (control: 
no phagocytosis) or $37^{\circ} \mathrm{C}$ (test) with FITC-labeled E. coli. Phagocytosis was halted by the addition of cold PBS, whereas cell surface-bound FITC was quenched by addition of $1 \%$ trypan blue solution. Unbound free bacteria were removed by washing in PBS, then erythrocytes were lysed and leukocytes were fixed using 1\% fix/lyse solution (Thermo Fisher Scientific). Cell DNA was counterstained by addition of propidium iodide (PI) in order to gate on immune cells before flow cytometry was performed, and 10,000 neutrophils were acquired. Median fluorescence intensity (MFI) of the control was subtracted from the test to give a final phagocytic value. The MFI corresponds to a cell-by-cell "amount' or phagocytic capacity of $E$. coli.

\section{Neutrophil ROS production}

ROS generation was assessed by luminol-amplified chemiluminescence as previously described [45]. Briefly, resting neutrophils $\left[1 \times 10^{5}\right.$ in $\mathrm{HBSS}+/+(+$ calcium + magnesium $\left.)\right]$ were dispensed into a 96-well, white, flat-bottomed plate (Corning, Corning, NY, USA) containing $1 \mu \mathrm{M}$ luminol (pH 7.3; Sigma-Aldrich). Cells were stimulated with $25 \mathrm{nM}$ phorbol 12-myristate 13-acetate (PMA) (test) or $\mathrm{HBSS}+/+$ [46] and immediately assessed for ROS generation at 1-minute intervals for 60 minutes using an Infinite 200 PRO plate reader (Tecan Life Sciences, Männedorf, Switzerland). Experiments were performed in triplicate, with ROS production measured as relative light units and calculated as the AUC.

Measurement of immune cell surface receptor expression Neutrophil surface receptor expression was assessed in whole blood. Briefly, $100 \mu \mathrm{l}$ of heparin-treated blood was dispensed into $5-\mathrm{ml}$ tubes and stored at $4{ }^{\circ} \mathrm{C}$ in the dark. Cells were stained with anti-CXCR2-phycoerythrin (anti-CXCR2-PE, clone 5E8-C7-F10; Thermo Fisher Scientific), anti-CD16-FITC (clone 3G8; BD Biosciences, San Jose, CA, USA), anti-CD11b-allophycocyanin (anti-CD11b-APC, clone ICRF44; BD Biosciences), anti-CD18-PE (clone 6.7; BD Biosciences), anti-Toll-like receptor 2 (TLR2)-Alexa Fluor 647 (clone 11G7; BD Biosciences), or anti-TLR4-APC (clone HTA-125; Thermo Fisher Scientific), or their relevant concentration-matched isotype controls for 60 minutes on ice in the dark. Following incubation, cells were washed twice in cold PBS, and erythrocytes were lysed and leukocytes were fixed using 1\% fix/lyse solution (Thermo Fisher Scientific). Following fixing, cells were washed twice and resuspended in $300 \mu \mathrm{l}$ of PBS for analysis by flow cytometry.

Monocyte surface receptor expression was assessed on freshly isolated PBMCs $\left(1 \times 10^{5}\right.$ cells $\left./ \mathrm{ml}\right)$. Cells were stained with CD14-Pacific Blue (clone TuK4; Thermo Fisher Scientific), CD16-FITC, TLR2-Alexa Fluor 647, TLR4-PE, anti-HLA-DR-PE-CF594 (clone G46-6; BD
Biosciences), or relevant isotype controls for 30 minutes at $4{ }^{\circ} \mathrm{C}$ in the dark. Postincubation cells were washed twice in $\mathrm{PBS} / 1 \% \mathrm{BSA}$, resuspended in $300 \mu \mathrm{l}$ of $\mathrm{PBS} / 1 \% \mathrm{BSA}$, and transferred to polypropylene fluorescence-activated cell sorting tubes for analysis by flow cytometry.

All flow cytometric analyses were conducted on a BD FACSCanto II (BD Biosciences) flow cytometer equipped with three lasers using the Duke Cancer Institute Core Facility, which maintained daily quality controls of the machine. Ten thousand neutrophils and 5000 monocytes were acquired for analysis. Data were analyzed using FCS Express 6 (De Novo Software, Glendale, CA, USA).

\section{Plasma analyses}

Samples were processed immediately for plasma and immune cell isolation, and relevant samples were stored at $-80{ }^{\circ} \mathrm{C}$ until analysis. All plasma analyses were completed by the Core Facilities within the Duke Molecular Physiology Institute. Plasma samples obtained following an overnight fast were analyzed for five cytokines and one acute-phase protein: CXCL-8, IL-6, IL-10, TNF- $\alpha$, IL-1 $\beta$, and C-reactive protein (CRP). Concentrations of cytokines were determined in duplicate using a human proinflammatory 5-plex sandwich immunoassay according to the manufacturer's instructions (Meso Scale Discovery, Rockville, MD, USA). High-sensitivity CRP was measured in duplicate using a commercially available ELISA (IBL International, Hamburg, Germany). The lower limits of detection (LLODs) were as follows: CXCL-8 (0.08 pg/ml), IL-6 (0.11 pg/ml), IL-10 (0.05 pg/ml), TNF- $\alpha$ (0.09 pg/ml), IL-1 $\beta \quad(0.03 \mathrm{pg} / \mathrm{ml})$, and CRP $(0.02 \mathrm{mg} / \mathrm{L})$. All samples had concentrations greater than the LLOD, with the exception of IL- $1 \beta$ with $77 \%$ of samples above the LLOD. Plasma concentrations of nonesterified fatty acid (NEFA) were assessed by an enzymatic colorimetric assay on a UniCel DxC600 Analyzer using the manufacturer's guidelines (Beckman Coulter Life Sciences, Indianapolis, IN, USA).

\section{Statistics}

All analyses were conducted using IBM SPSS Statistics version 23.0 software (IBM, Armonk, NY, USA), and all data are presented as mean \pm SD unless otherwise stated. The primary outcome of the study was change in disease activity, and the secondary outcome was change in $\mathrm{VO}_{2 \text { peak. }}$ We initially powered the study to detect a $5 \%$ change in $\mathrm{VO}_{2 \text { peak }}$ based on preliminary data in diabetic adults as in our previous studies [25]. The sample size for change in $\mathrm{VO}_{2 \text { peak }}$ was five participants. Following completion of our analysis of these five participants, we powered the remainder of the study on change in disease activity. Sample size calculations suggested a total of 12 participants would be needed to detect a significant $16 \%$ reduction $(80 \%$ power) in disease activity. Normality was 
assessed using Kolmogorov-Smirnov analysis; natural logarithmic transformation of distributed variables violating normality was completed. Pairwise comparisons of variables were completed using paired $t$ tests. Bivariate correlations were conducted between changes in fitness, body composition, inflammatory markers, and immune functions to tease out associations. Statistical significance was accepted as $p \leq 0.05$.

\section{Results}

\section{HIIT effects on health and RA disease activity}

Information on clinical characteristics, disease activity, and medication use is shown in Table 1. Participants were receiving a range of rheumatic medications, including immunosuppressive, anti-inflammatory, and steroidal drugs, and these medications were maintained during the study. Mean arterial blood pressure $(p=0.044)$, resting heart rate $(p=0.009)$, and feelings of depression (HADS questionnaire; $p=0.031$ ) were all reduced following training. Disease activity improved by a clinically significant $38 \%$, corresponding to a mean disease activity reduction from moderate to low (both $p=0.001$ ). There was a mean $58 \%$ reduction in $\operatorname{ESR}(p=0.02)$, but no changes for CRP $(p>0.05)$. Reductions in disease activity were mediated primarily by small reductions in the numbers of swollen and tender joints, improved global health, and in the case of DAS28 $8_{\text {ESR }}$ owing to a significant reduction in ESR. There were no HIIT-induced changes in body composition, self-reported disability (HAQ-DI), pain, or anxiety (all $p>0.05$ ).

\section{HIIT effects on cardiorespiratory fitness and physical function}

Table 2 shows that 10 weeks of HIIT resulted in a $9 \pm 4 \%$ increase in both relative $(p<0.001)$ and absolute $(p<0.001)$ cardiorespiratory fitness (range, $0-15 \%$ ), similar to our previous observations with HIIT in younger healthy adults [25]. Body composition, including body mass index (BMI) and body fat percentage, were unchanged following training (both $p>0.05$ ). Because physical functioning is generally impaired in patients with RA, we sought to determine function in a number of ways. Assessment of habitual physical activity was determined by the IPEQ and the SBAS. The IPEQ $(p=0.788)$ and SBAS $(p=0.096)$ results suggested that participants were no more active outside the prescribed training program than in it. As expected, because the training used a walking protocol, grip strength was unchanged following training $(p>0.05)$. However, there were significant improvements in lower limb function, as indicated by an $8 \pm 4 \%$ reduction in time needed to walk $400 \mathrm{~m}(p=0.001)$ and an $11 \pm 12 \%$ increase in the number of chair-stands completed over 30 seconds $(p=0.035)$.
HIIT effects on systemic inflammation, white blood cell counts and metabolic health

In order to determine if exercise training had any effect on systemic inflammation and measures of glucose and fatty acid control, we assessed a small panel of pro- and anti-inflammatory markers, white blood cell counts, glucose, insulin, and nonesterified fatty acid (NEFA) concentrations, (Table 3). No changes were observed for IL-1 $\beta$, IL-6, CXCL-8, TNF- $\alpha$, or the anti-inflammatory cytokine IL-10 (all $p>0.05$ ). There were no traininginduced changes in complete blood cell count 16-24 hours after the last exercise bout, suggesting minimal to no influence of the final acute bout (all $p>0.05$ ). Similarly, fasting glucose, insulin, and NEFAs did not change (all $p>0.05$ ).

\section{HIIT effects on innate immune function}

Improved neutrophil migration and bactericidal function In response to 10 weeks of HIIT, migration of isolated RA peripheral blood neutrophils toward the chemokine CXCL-8 improved. A doubling of the chemotactic accuracy (chemotactic index; $p=0.003 ; \mathrm{CI},-0.3,-0.1$; $d=1.1$ ) was driven by improved directional speed (chemotaxis, $p=0.044$; CI, $-1.2,-0.02 ; d=0.66$ ), with no effect on overall speed (chemokinesis, $p=0.272$; CI, $-1.5,0.5$; $d=0.33$ ) (Fig. 1a-c). Following HIIT, neutrophils phagocytized more FITC-labeled E. coli (Fig. 2a and b) ( $p=0.03$; CI, $-2884,-87$; $d=0.71)$. Accompanying the improved phagocytosis, training enhanced neutrophil PMA-stimulated ROS production (Fig. 2d) $(p<0.001$; CI, $-69,964,-25,737 ; d=1.4$ ). No differences were observed for expression of the relevant surface receptors, which could account for these changes, namely the CXCL-8 receptor CXCR2 (Fig. 2e) ( $p=0.865$; CI, -105 , 90; $d=0.06$ ), the ICAM-1 receptors CD11b/CD18 (Fig. 2f) $(p=0.404 ; \mathrm{CI},-96,207 ; d=0.27)$, TLR4 (Fig. 2g) ( $p=0.929$; CI, $-158,147 ; d=0.03$ ), or the Fcy receptor CD16 (Fig. 2h) $(p=0.300 ; \mathrm{CI},-571,1652 ; d=0.35)$. Taken together, these data suggest that HIIT improves neutrophil migratory accuracy and bactericidal functions in patients with stable RA.

Shifts from pro- to anti-inflammatory monocyte phenotypes Depending on their expression of CD14 and CD16, monocytes can be considered to be composed of three distinct populations (Fig. 3a). Following 10 weeks of HIIT, there were significant reductions in the frequency of total CD16-expressing proinflammatory monocytes (Fig. 3b) ( $p=0.002 ; \mathrm{CI}, 2.5,8.4 ; d=1.3$ ) owing to reduced frequency of both intermediate CD14 $4^{\text {bright }} / \mathrm{CD} 16^{\text {positive }}$ (Fig. 3c) $(p=0.009$; CI, 0.9, 4.7; $d=1.04)$ and nonclassical CD14 $4^{\mathrm{dim}} / \mathrm{CD} 16^{\text {bright }}$ (Fig. 3d) ( $p=0.045$; CI, $0.1,5.3 ; d=0.73)$ monocytes. On intermediate monocytes, there was reduced expression of TLR2 (Fig. 3e) $(p=0.005$; CI, 182, 738; $d=1.27)$, TLR4 (Fig. 3f) 
Table 1 Before and after high-intensity interval training values, Cls, and effect sizes for clinical characteristics, health and disease activity, and medication use

\begin{tabular}{|c|c|c|c|c|c|}
\hline & Pre-HIIT & Post-HIIT & 95\% Cl (lower, upper) & $p$ Value & Effect size $(d)$ \\
\hline Age, yr & $64 \pm 7$ & & & & \\
\hline Sex, M/F & $1 / 11$ & & & & \\
\hline \multicolumn{6}{|l|}{ Clinical characteristics } \\
\hline $\mathrm{BMI}, \mathrm{kg} / \mathrm{m}^{2}$ & $27.4 \pm 9.3$ & $27.7 \pm 9.8$ & $(-0.6,0.1)$ & 0.191 & 0.40 \\
\hline Body fat, $\%$ & $36.6 \pm 11.6$ & $37.3 \pm 11.2$ & $(-2.1,0.7)$ & 0.294 & 0.32 \\
\hline \multicolumn{6}{|l|}{ Blood pressure, $\mathrm{mmHg}$} \\
\hline Systolic & $136 \pm 14$ & $127 \pm 14$ & $(-0.8,17.3)$ & 0.070 & 0.58 \\
\hline Diastolic & $73 \pm 12$ & $69 \pm 8$ & $(-2.7,8.1)$ & 0.064 & 0.60 \\
\hline Mean arterial pressure & $94 \pm 11$ & $89 \pm 9$ & $(0.2,10.3)$ & 0.044 & 0.66 \\
\hline Resting heart rate, beats/min & $69 \pm 8$ & $64 \pm 8$ & $(1.5,8.3)$ & 0.009 & 0.91 \\
\hline $\mathrm{HbA} 1 \mathrm{c}, \%$ & $5.6 \pm 0.5$ & $5.6 \pm 0.4$ & $(-0.1,0.2)$ & 0.898 & 0.04 \\
\hline \multicolumn{6}{|l|}{ Health and disease activity } \\
\hline HAQ-DI & $0.42 \pm 0.35$ & $0.39 \pm 0.47$ & $(-0.1,0.2)$ & 0.614 & 0.16 \\
\hline Pain & $28.7 \pm 29.8$ & $20.3 \pm 21.5$ & $(-7.3,24.1)$ & 0.262 & 0.34 \\
\hline Anxiety & $4.1 \pm 2.6$ & $3.5 \pm 2.8$ & $(-0.9,2.0)$ & 0.393 & 0.25 \\
\hline Depression & $3.8 \pm 2.5$ & $2.4 \pm 1.9$ & $(0.1,2.5)$ & 0.031 & 0.71 \\
\hline Swollen joints, $n$ & $4.3 \pm 3.8$ & $1.9 \pm 1.7$ & $(0.6,4.2)$ & 0.013 & 0.85 \\
\hline Tender joints, $n$ & $4.3 \pm 7$ & $1.9 \pm 2.8$ & $(-0.8,5.4)$ & 0.062 & 0.48 \\
\hline Global health, mm & $31.5 \pm 15.7$ & $22.6 \pm 19.2$ & $(0.6,17.2)$ & 0.037 & 0.85 \\
\hline DAS28 ESR & $3.1 \pm 1.6$ & $2.3 \pm 1.2$ & $(0.4,1.2)$ & 0.001 & 1.23 \\
\hline DAS28 $8_{C R P}$ & $3.1 \pm 1.2$ & $2.4 \pm 1$ & $(0.3,1.0)$ & 0.001 & 1.26 \\
\hline $\mathrm{ESR}, \mathrm{mm} / \mathrm{h}$ & $10.5 \pm 11.9$ & $7 \pm 8.8$ & $(0.04,6.5)$ & 0.023 & 0.65 \\
\hline $\mathrm{CRP}, \mathrm{mg} / \mathrm{L}$ & $2.7 \pm 3.5$ & $2.2 \pm 3.1$ & $(-0.6,1.6)$ & 0.322 & 0.30 \\
\hline \multicolumn{6}{|l|}{ Medication use, $n(\%)$} \\
\hline Infliximab & $2(17 \%)$ & & & & \\
\hline Adalimumab & $2(17 \%)$ & & & & \\
\hline Methotrexate & $6(50 \%)$ & & & & \\
\hline Leflunomide & $1(8 \%)$ & & & & \\
\hline Sulfasalazine & $2(17 \%)$ & & & & \\
\hline Tofacitinib & $1(8 \%)$ & & & & \\
\hline Hydroxychloroquine & $4(33 \%)$ & & & & \\
\hline NSAID & $8(67 \%)$ & & & & \\
\hline Prednisone & $2(17 \%)$ & & & & \\
\hline
\end{tabular}

Abbreviations: HIIT High-intensity interval training, HbA1c Hemoglobin A1c, BMI Body mass index, HAQ-DI Health Assessment Questionnaire Disability Index DAS28 Disease Activity Score in 28 joints, ESR Erythrocyte sedimentation rate, CRP C-reactive protein, NSAID Nonsteroidal anti-inflammatory drug Data are mean \pm SD

$(p=0.026 ;$ CI, 20, 239; $d=0.90)$ and HLA-DR (Fig. 3g) $(p=0.037 ; \mathrm{CI}, \quad 306,7528 ; d=0.83)$. On classical $\mathrm{CD} 14^{\text {bright }} / \mathrm{CD} 16^{\text {negative }}$ and nonclassical monocytes, there were no differences for TLR2, TLR4, or HLA-DR expression. Additionally, there was a trend toward an increased percentage of classical monocytes at the expense of proinflammatory, $\mathrm{CD}_{16}{ }^{+}$monocytes $(p=0.062$; CI, $-5.7,0.2$; $d=0.68)$. A greater percentage of classical monocytes may have accounted for increased phagocytosis of FITC-labeled E. coli following training (Fig. $3 \mathrm{~h})$ ( $p=0.02$; CI, $-3260,-340 ; d=0.83)$. Taken together, these data suggest that HIIT improves the balance of inflammatory pathologically related CD16-expressing monocytes to CD16 negative monocytes. 
Table 2 Before and after high-intensity interval training values, Cls, and effect sizes for cardiorespiratory fitness and physical function

\begin{tabular}{|c|c|c|c|c|c|}
\hline & Pre-HIIT & Post-HIIT & 95\% Cl (lower, upper) & $p$ Value & Effect size $(d)$ \\
\hline \multicolumn{6}{|l|}{ Cardiorespiratory fitness } \\
\hline $\mathrm{VO}_{2 \text { peak, }} \mathrm{L} / \mathrm{min}$ & $1.75 \pm 0.4$ & $1.89 \pm 0.4$ & $(-3.0,-1.8)$ & $<0.001$ & 2.86 \\
\hline $\mathrm{VO}_{2 \text { peak, }} \mathrm{ml} / \mathrm{kg} / \mathrm{min}$ & $25.0 \pm 6.6$ & $27.1 \pm 7.0$ & $(-0.2,-0.13)$ & $<0.001$ & 3.19 \\
\hline \multicolumn{6}{|l|}{ Physical function } \\
\hline \multicolumn{6}{|c|}{ Physical activity questionnaires } \\
\hline IPEQ & $30.4 \pm 24.6$ & $28.8 \pm 16.7$ & $(-11.2,14.4)$ & 0.788 & 0.08 \\
\hline SBAS & $2.5 \pm 0.5$ & $2.9 \pm 1.0$ & $(-0.9,0.1)$ & 0.096 & 0.52 \\
\hline Grip strength, kg & $18.3 \pm 7.2$ & $19.0 \pm 8.1$ & $(-1.7,0.3)$ & 0.166 & 0.43 \\
\hline Berg Balance Score & $54.4 \pm 4.0$ & $54.7 \pm 3.2$ & $(-1.2,0.7)$ & 0.555 & 0.18 \\
\hline TUG, $\mathrm{s}$ & $8.8 \pm 1.8$ & $8.4 \pm 1.9$ & $(-0.1,0.1)$ & 0.084 & 0.54 \\
\hline 400-m walk test, s & $251 \pm 62$ & $233 \pm 51$ & $(9.0,27.4)$ & 0.001 & 1.30 \\
\hline 30-s chair stands, $n$ & $14 \pm 4$ & $17 \pm 5$ & $(-4.0,-0.2)$ & 0.035 & 0.70 \\
\hline
\end{tabular}

Abbreviations: HIIT High-intensity interval training, IPEQ Incidental and Planned Exercise Questionnaire, SBAS Stanford Brief Activity Survey, TUG Timed Up and Go, $\mathrm{VO}_{2 \text { peak }}$ Aerobic capacity

Data are mean \pm SD

Correlations on disease activity, fitness, body composition, immune function, and inflammation

To determine relationships between changes in our outcome variables, we conducted correlation analyses between changes in measures of disease activity, fitness, body composition, immune function, and inflammatory markers. The only significant correlation observed was between changes in relative $\mathrm{VO}_{2 \text { peak }}$ and IL-10, with increasing fitness associated with higher concentrations of IL-10 (rho $=0.653 ; p=0.029$ ).

\section{Discussion}

Ten weeks of high-intensity interval walking above exercise intensity health guidelines was associated with

Table 3 Before and after high-intensity interval training values, Cls, and effect sizes for systemic inflammation, white blood cell counts, glucose, insulin and insulin resistance, and nonesterified fatty acid concentrations

\begin{tabular}{|c|c|c|c|c|c|}
\hline & Pre-HIIT & Post-HIIT & 95\% Cl (lower, upper) & $p$ Value & Effect size $(d)$ \\
\hline \multicolumn{6}{|l|}{ Systemic inflammation } \\
\hline $\mathrm{IL}-1 \beta, \mathrm{pg} / \mathrm{ml}$ & $0.12 \pm 0.13$ & $0.12 \pm 0.11$ & $(-0.04,0.06)$ & 0.788 & 0.07 \\
\hline $\mid \mathrm{L}-6, \mathrm{pg} / \mathrm{ml}$ & $1.06 \pm 0.89$ & $1.43 \pm 2.02$ & $(-1.6,0.8)$ & 0.519 & 0.19 \\
\hline CXCL-8, pg/ml & $9.69 \pm 6.97$ & $10.3 \pm 10.0$ & $(-3.3,2.2)$ & 0.655 & 0.13 \\
\hline IL-10, pg/ml & $0.5 \pm 0.4$ & $0.5 \pm 0.3$ & $(-0.1,0.1)$ & 0.912 & 0.03 \\
\hline TNF-a, pg/ml & $2.21 \pm 0.96$ & $2.31 \pm 0.96$ & $(-0.5,0.3)$ & 0.570 & 0.17 \\
\hline \multicolumn{6}{|l|}{ White blood cells, $\times 10^{6} / \mathrm{L}$} \\
\hline Total count & $5.6 \pm 1.1$ & $5.8 \pm 1.2$ & $(-0.6,0.2)$ & 0.322 & 0.32 \\
\hline Neutrophils & $2.8 \pm 0.8$ & $2.9 \pm 0.9$ & $(-0.5,0.1)$ & 0.217 & 0.40 \\
\hline Lymphocytes & $2.0 \pm 0.6$ & $2.1 \pm 0.6$ & $(-0.4,0.2)$ & 0.623 & 0.15 \\
\hline Monocytes & $0.6 \pm 0.2$ & $0.6 \pm 0.2$ & $(-0.1,0.1)$ & 0.676 & 0.12 \\
\hline Eosinophils & $0.3 \pm 0.3$ & $0.2 \pm 0.1$ & $(-0.02,0.2)$ & 0.108 & 0.53 \\
\hline Neutrophils: lymphocytes & $1.5 \pm 0.6$ & $1.5 \pm 0.5$ & $(-0.4,0.3)$ & 0.810 & 0.07 \\
\hline \multicolumn{6}{|l|}{ Glucose, insulin, and NEFA } \\
\hline Fasting glucose (mg/dl) & $90.9 \pm 7.7$ & $93.1 \pm 8.3$ & $(-4.8,0.5)$ & 0.098 & 0.55 \\
\hline Fasting insulin, $\mu \mathrm{lU} / \mathrm{ml}$ & $13 \pm 4.9$ & $12 \pm 4.8$ & $(-0.8,2.7)$ & 0.264 & 0.34 \\
\hline HOMA-IR & $1.67 \pm 0.64$ & $1.56 \pm 0.62$ & $(-0.1,0.3)$ & 0.258 & 0.34 \\
\hline $\mathrm{NEFA}, \mathrm{mmol} / \mathrm{L}$ & $0.55 \pm 0.37$ & $0.60 \pm 0.36$ & $(-0.2,0.1)$ & 0.412 & 0.24 \\
\hline
\end{tabular}

Abbreviations: HIIT High-intensity interval training, IL Interleukin, CXCL Chemokine C-X-C motif ligand, TNF- $a$ Tumor necrosis factor-a, HOMA-IR Homeostatic model of assessment of insulin resistance, NEFA Nonesterified fatty acids

Data are mean \pm SD 


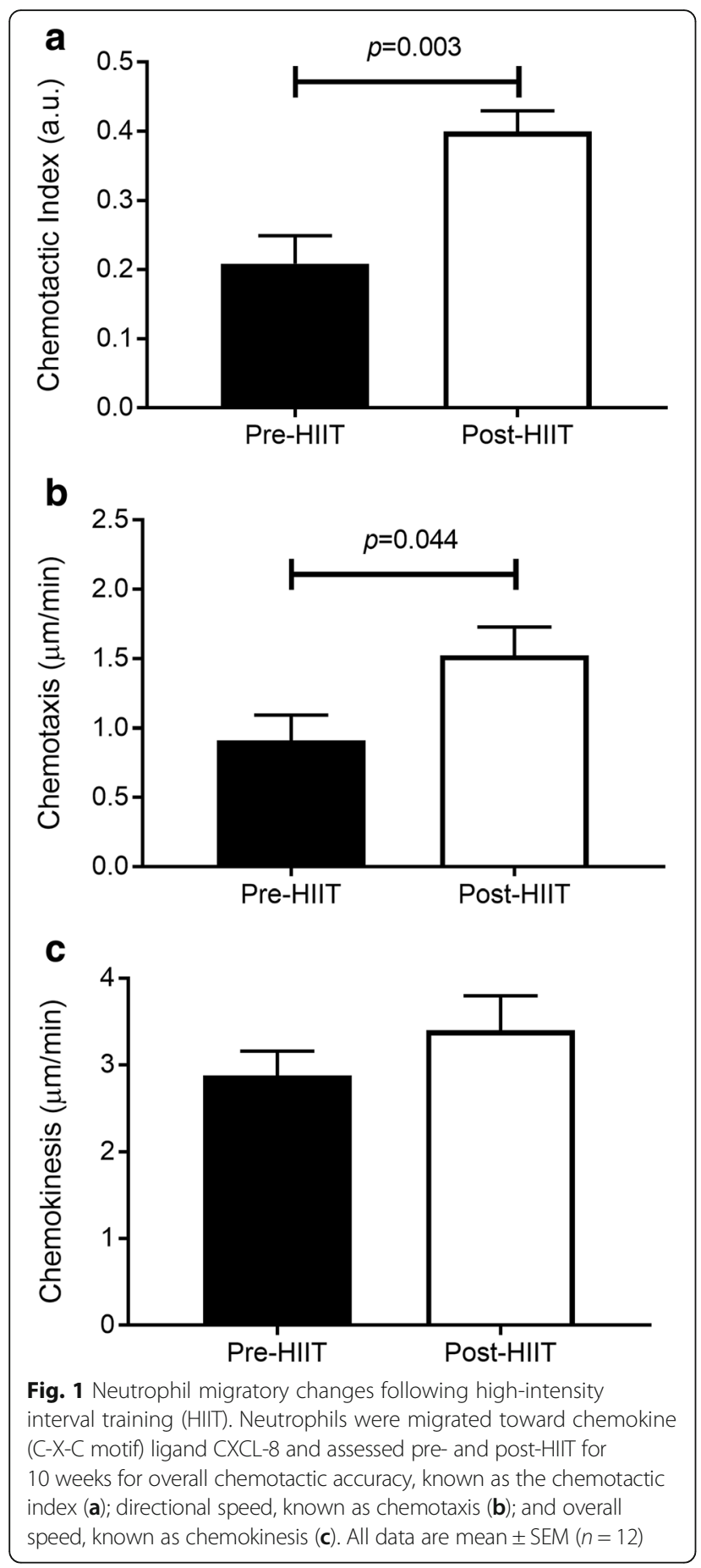

reduced disease activity, increased aerobic capacity, and improved antibacterial innate immune function in patients with RA. Without a sufficient control group, it is unclear exactly how much HIIT walking contributed to these changes. Changes in disease activity were associated with reductions in ESR and joint swelling and with increased global perceived health. Furthermore, there were associations with changes in innate immune function, including neutrophil chemotaxis, bacterial phagocytosis and ROS production, improved monocyte bacterial phagocytic capacity, and a shift toward a classical CD $14^{\text {bright }} / \mathrm{CD} 16^{\text {negative }}$ monocyte phenotype. These functional immune improvements potentially enhance antibacterial function, reducing the risk of infection.

\section{Disease activity, physical fitness, and function in RA}

There is a significant body of literature showing that physical activity and exercise training are safe for patients with RA and do not aggravate disease activity [47-50]. Although studies have suggested that individual components of disease activity can be improved, few have reported actual lowering of disease activity scores [48]. In the present study, using a composite, wellvalidated disease activity score including assessment of 28 joints for tenderness and swelling, perceived global health, and inflammatory markers (ESR or CRP), overall disease activity was reduced by $38 \%$ following training. Reductions appeared to be driven primarily by improvements in joint health, as determined by swollen and tender joints, improved participant feelings of health, and reduced ESR. This reduction was sufficient to reclassify participants on average from moderate to low disease activity. Such a reduction is unlikely to be observed without exercise training; however, because we did not have a control group, this cannot be discounted.

It is not completely clear what accounted for our observed disease activity improvements. One possibility is that by using higher intensities and grade elevations, HIIT had effects on lower extremity strength. Strength improvements were evident by improved sit-to-stand ability. Walking was at faster speeds than individual participants' normal speeds, as evidenced by comparisons with calculated speeds from both the TUG and 400-m walk speed (data not shown) tests. As such, the only other exercise intervention studies to show disease score reductions incorporated resistance training, suggesting a link between muscle strength and disease activity [51-55]. In light of this, we now add to the literature that HIIT walking is likely associated with improved disease activity. Further, as compared with other aerobic exercise interventions, no significant adverse events and a $9 \%$ increase in cardiorespiratory fitness were observed, suggesting that our program is safe and effective in improving cardiorespiratory fitness [30, 32].

\section{HIIT and immune function in RA}

Although RA is considered an inflammatory disease characterized by increased systemic and joint inflammation, the pathology of the inflammation is due primarily to a deregulated immune system. Specifically, there is a growing body of evidence suggesting that habitual physical activity and exercise training promote enhanced 
a

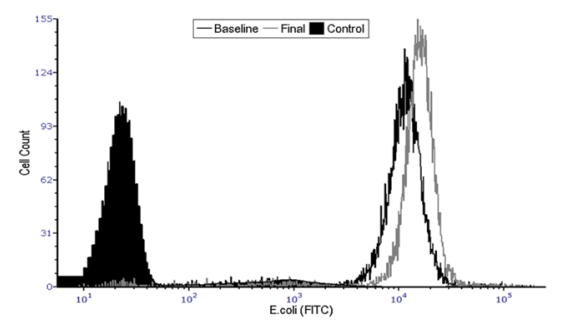

C

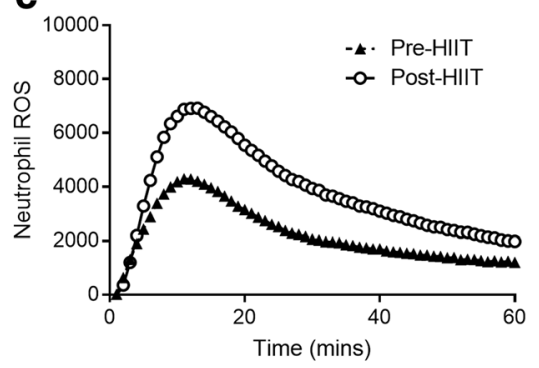

e
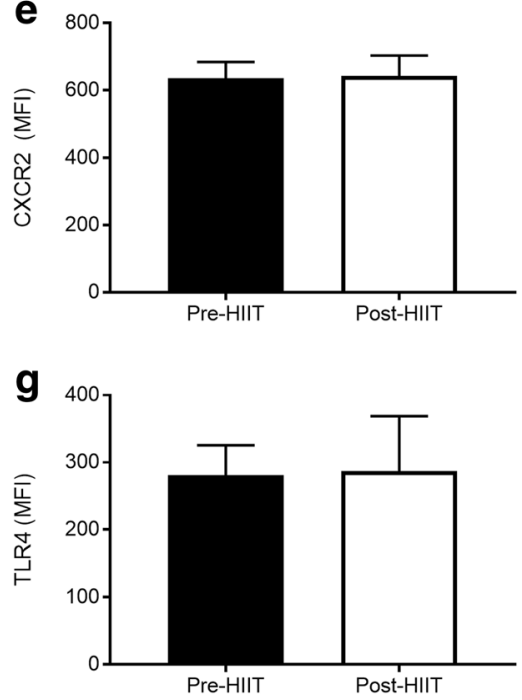

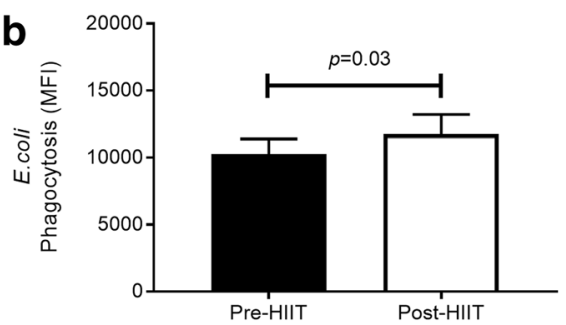

d

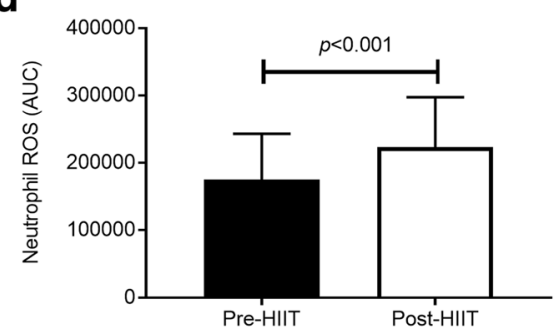

f

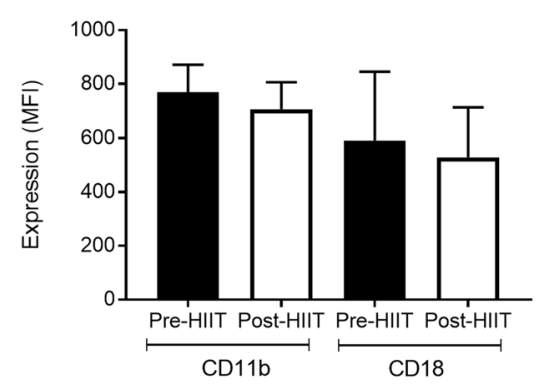

h

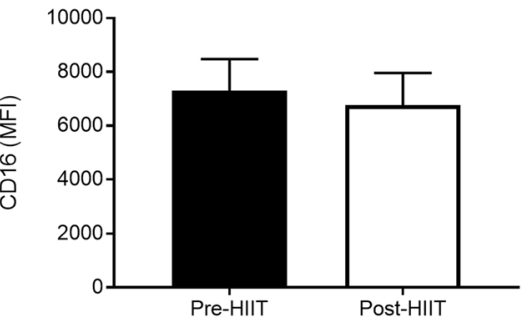

Fig. 2 Neutrophil changes in response to 10 weeks of high-intensity interval training (HIIT). Representative flow cytometric histograms showing (a) improved neutrophil phagocytosis of Escherichia coli, (b) phagocytic capacity, (c) representative diagram of the kinetic phorbol 12-myristate 13-acetate-stimulated ROS production, and (d) ROS generation (AUC) and neutrophil surface receptor expression pre- and post-HIIT training for (e) CXC chemokine receptor 2 (CXCR2), (f) cluster of differentiation 11b (CD11b)/CD18, (g) Toll-like receptor 4 (TLR4), and (h) CD16. All data are mean \pm SEM $(n=12)$. MFI Median fluorescence intensity

immune function in both adaptive and innate cells [56]. Although exercise can improve immune function in age-comparable healthy adults [57], few studies have assessed the effects of exercise training on immune function in patients with RA. Because patients with RA are at increased risk of opportunistic infections owing to impaired and reduced immune responses, promoting better immune function likely reduces these risks. Very few studies have assessed immune responses to exercise in RA, with no changes in lymphocyte proliferation or natural killer (NK) cell tumor cell cytotoxicity [31, 58], suggesting that exercise may not enhance primary cell functions. However, because patients with RA are at increased risk of infection and require optimal neutrophil and monocyte function, we were interested in whether HIIT would improve antibacterial functions of innate immune cells.

When compared with healthy age-matched control subjects, patients with stable RA have similar neutrophil migration $[59,60]$. Although increased neutrophil migration from the blood to the synovium is observed in the early stages of RA, improved chemotactic accuracy should benefit older patients with RA [4, 59, 61], specifically because poor chemotactic accuracy results in more aberrant tissue damage and elongated resolution of infection and increased inflammatory insult. In a study 
a

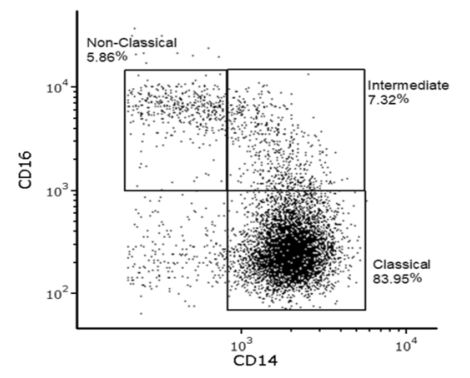

C
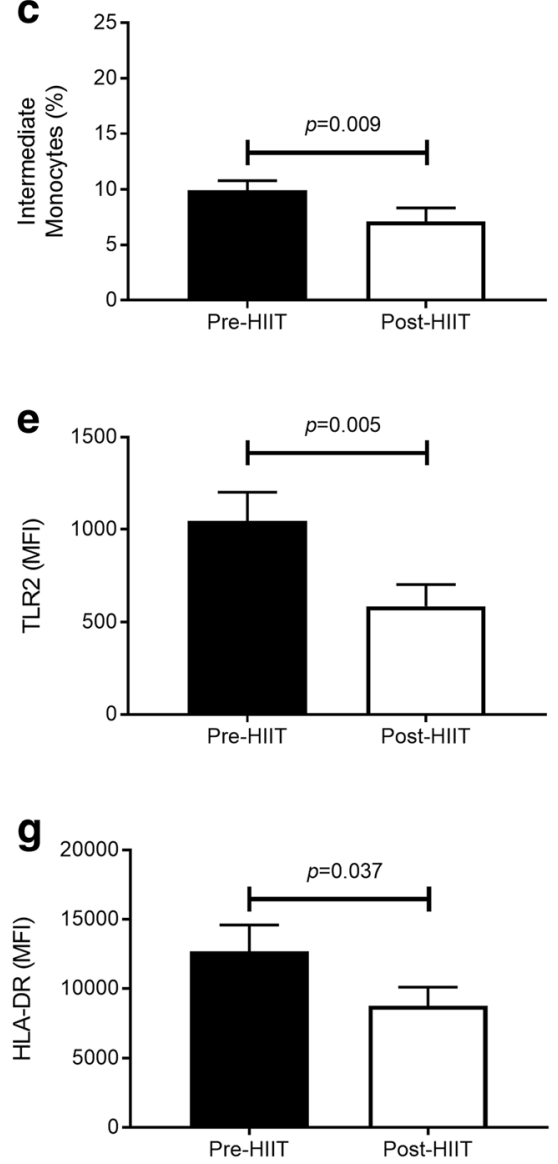

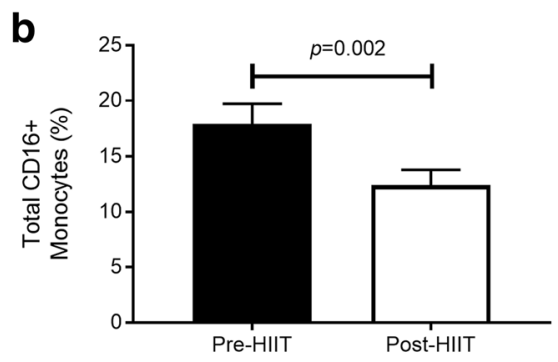

d

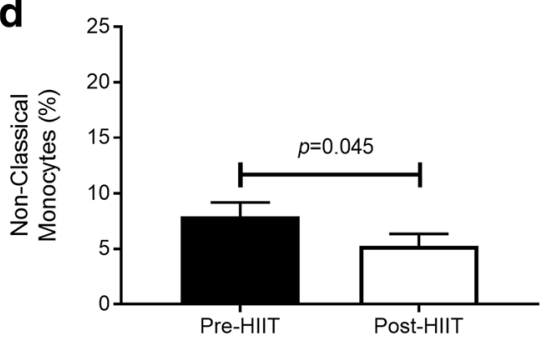

f
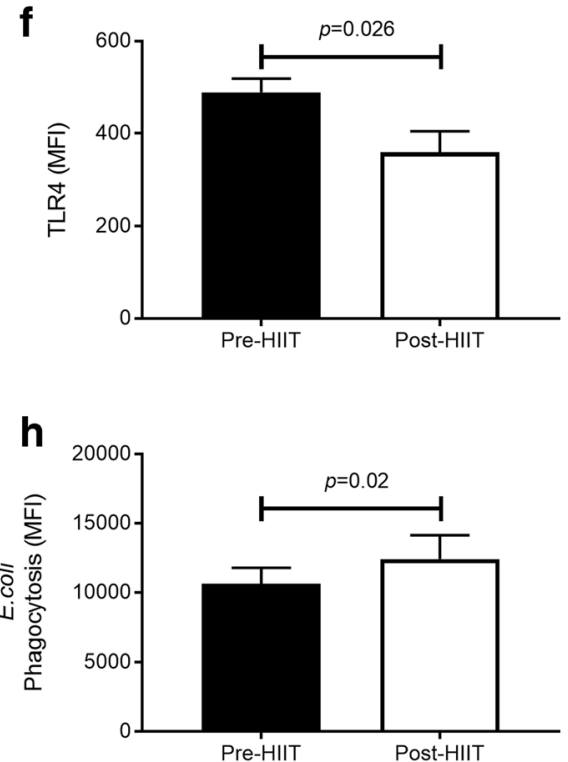

Fig. 3 Monocyte changes in response to 10 weeks of high-intensity interval training (HIIT). a Representative forward vs. side scatter of cluster of differentiation 16 (CD16) expression on CD14 $4^{+}$monocytes. Frequency of (b) total CD16-expressing monocytes, (c) intermediate monocytes, and (d) nonclassical monocytes pre- and post-HIIT training. Surface expression of (e) Toll-like receptor 2 (TLR2), (f) TLR4, and (g) human leukocyte antigen (HLA)-DR on intermediate monocytes pre- and post-HIIT training. $\mathbf{h}$ Phagocytosis of fluorescein isothiocyanate-labeled Escherichia coli pre- and post-HIIT training. All data are mean \pm SEM $(n=12)$. MFI Median fluorescence intensity

of healthy older adults, those who completed more steps per day $(10,370 \pm 3221$ vs. $4746 \pm 1351)$ had better neutrophil chemotactic accuracy, suggesting that exercise training may improve neutrophil migratory capacity [20]. Neutrophil migration was also improved in women with hypertension older than 60 years of age following 6 months of exercise training and in young sedentary participants following 2 months of cycling training $[62,63]$. We add to the literature by showing, for the first time to our knowledge, that HIIT is associated with improved neutrophil migration toward a common chemokine produced during infectious episodes in older adults with stable RA.

Along with migration, other critical primary neutrophil functions include phagocytosis and ROS killing of pathogens. Although excessive ROS production in the synovium contributes to disease pathology, it is unclear what role blood neutrophil ROS play in RA [5, 64]. 
Compared with healthy control subjects, in RA blood neutrophil ROS are either similar or reduced [5, 7], whereas reduced ROS with aging is a classic hallmark of neutrophil dysfunction [65]. Similarly, there is no clear consensus regarding whether peripheral blood neutrophil phagocytosis is altered in RA [66-70]. Paino and colleagues suggested that phagocytosis of $E$. coli is greater in older women with RA than in matched control subjects [67], whereas Turner and colleagues suggested that phagocytosis is reduced in RA [68]. Similarly to our previous HIIT study in healthy adults [25], we show increased stimulated ROS production and increased bacterial phagocytosis following exercise training, suggesting an enhanced ability of neutrophils to kill bacterial pathogens. Furthermore, in agreement with previous studies, functional improvements are not associated with changes in relevant cell surface receptor expression, suggesting intrinsic cellular changes [4, 20, 25].

Neutrophil dysfunction is associated with increased infection risk, and so is monocyte/macrophage dysfunction. Further, with RA and aging, monocytes adopt more of an inflammatory phenotype with the potential to add to the inflammatory milieu. There are three subtypes of blood monocytes with distinct inflammatory and functional characteristics dependent on their expression of CD16 [71-73]. In addition to CD16, TLR2 and TLR4 are typically expressed more on monocytes/macrophages in RA $[10,11,74]$. In healthy sedentary adults, HIIT can reduce expression of CD16, TLR2, and TLR4 while increasing monocyte phagocytosis of $E$. coli $[25,28]$. We add that in older patients with stable RA, HIIT is associated with increased phagocytic capacity of $E$. coli as well as reductions in the total percentage of CD16-expressing monocytes through reductions in the percentage of both intermediate and nonclassical monocytes. Furthermore, the expression of TLR2, TLR4, and HLA-DR was reduced on the intermediate proinflammatory monocytes, suggesting a reduced proinflammatory phenotype.

\section{HIIT and inflammation in RA}

RA medications are aimed at controlling inflammation [75]. Whether exercise training can reduce systemic inflammation in RA is an open question [18-20, 76]. It is likely that exercise has clinically or pathologically meaningful anti-inflammatory effects primarily in individuals with high, uncontrolled inflammation or when exercise is associated with reductions in body fat. The anti-inflammatory effects of exercise training have been reviewed extensively; yet, the mechanisms are still unclear. Long-term training likely exerts most of its anti-inflammatory effects by changing adipocyte mass and function [18]. In the present study, participants had well-controlled stable RA, were taking a range of anti-inflammatory medications, and had minimal nonsignificant changes in body fat; thus, we were not surprised to observe a lack of meaningful changes in systemic concentrations of CRP, IL-1 $\beta$, IL-6, CXCL-8, IL-10, or TNF- $\alpha$. These findings are similar to other aerobic-based exercise programs, suggesting that aerobic training without weight loss is insufficient to reduce most inflammatory markers in patients with RA, even those with well-controlled RA [30]. However, in agreement with studies using resistance training, we observed a significant reduction in the inflammatory marker ESR [51]. It is unclear on the basis of our study whether the changes in joint health, as determined by swollen and tender joint count reductions, were associated with reduced joint-specific inflammation. It is possible that joint health improved because of fluid shifts induced by mechanical loading; however, a number of the healthy joints were observed in the hands and arms. To determine relationships with training and inflammation, we conducted correlations between changes in fitness, body fat, and the aforementioned cytokines. No relationships were observed, other than increases in peak $\mathrm{VO}_{2}$ being associated with increasing concentrations of the anti-inflammatory cytokine IL-10. Future work aimed at understanding relationships between systemic and joint inflammation and exercise training may help illuminate this finding.

\section{Limitations and future directions}

Our study has limitations. A lack of an age-matched healthy control group does not allow us to state whether immune function was altered in our study group as a result of their disease. However, a control group in such a population is difficult to select because patients with RA are often more sedentary than healthy control subjects, their immune system shows signs of premature aging, and immune-modifying medications complicate immune function comparisons. In light of this, we could have recruited an RA nonexercise control group in order to show that changes were in fact due to the intervention and not dependent on time. However, a previous exercise study with an RA control group showed that 8 weeks of training was sufficient to increase cardiorespiratory fitness in the exercise group only. Subsequently, the control group did not have changes in immune cell proliferation, NK cell cytotoxicity, or inflammatory cytokine concentrations, suggesting that time alone does not change fitness or alter the immune system and inflammation in patients with RA [31]. Therefore, we are confident that the changes we observed were due to the exercise intervention and not to time alone.

Although this study was a pilot, considerations should be acknowledged regarding the population studied as well as the generalizability and feasibility of a larger, broader RA population to complete such an intervention. Our participants, although representative of our local RA population, were younger and had lower 
disease activity than much of the larger national RA demographics. As such, it is unclear whether such an intervention would be feasible and provide similar results in an older group with higher disease activity. Further, this was a supervised, structured exercise intervention, which would limit many individuals who are unable to access such expertise and gym facilities. Future studies should address a broader RA population and determine whether this program is feasible and can be transferred to a community- or home-based setting.

Further, we are confident that a significant portion of the effects was due to the intervention and that participants were not engaging in extra exercise outside our study design. Hower, we cannot be certain this did not occur. Although we used validated questionnaires, compared to quantifiable accelerometer data, questionnaires have inherent reliability issues.

The mechanisms by which exercise training in RA modifies disease activity and joint health or how changes in immune function can contribute are unclear. There is the potential that exercise training can improve immune-mediated joint health by moving dysfunctional cells out of the joint, allowing for replacement with healthier immune cells. Although this hypothesis remains untested in RA, animal exercise models suggest that exercise is associated with a redistribution of immune cells between tissues [77, 78]. Therefore, to elucidate the mechanisms associated with joint improvements, disease activity, and immune function, researchers in future studies should try to compare systemic exercise effects with those in the synovial fluid and local joint tissue.

\section{Conclusions}

We observed, for the first time to our knowledge, that 10 weeks of high-intensity interval walking in patients with stable RA was associated with clinically meaningful reductions in disease activity. Improvements in disease activity were accompanied by changes in immune cell function indicative of improved innate immunity and reduced risk of opportunistic bacterial infection. Our data suggest that HIIT walking could be an efficient, tolerable, and highly effective intervention to augment disease activity and improve overall health in patients with RA.

\footnotetext{
Abbreviations

APC: Allophycocyanin; BMI: Body mass index; BSA: Bovine serum albumin; CD: Cluster of differentiation; CRP: C-reactive protein; CXCL: Chemokine (C-X-C motif) ligand; CXCR: CXC chemokine receptor; DAS28: Disease Activity Score in 28 joints; ELISA: Enzyme-linked immunosorbent assay; ESR: Erythrocyte sedimentation rate; FITC: Fluorescein isothiocyanate; HAQ-DI: Health Assessment Questionnaire Disability Index; HADS: Hospital Anxiety and Depression Scale; HbA1c: Hemoglobin A1c; HBSS: Hanks' balanced salt solution; HIIT: High-Intensity Interval Training; HLA-DR: Human leukocyte antigen-DR; HOMA-IR: Homeostasis model assessment of insulin resistance; ICAM-1: Intercellular adhesion molecule 1; IL: Interleukin; IPEQ: Incidental and Planned Exercise Questionnaire; LLOD: Lower limit of
}

detection; MFI: Median fluorescence intensity; NEFA: Nonesterified fatty acids; NK: Natural killer cell; PBMC: Peripheral blood mononuclear cell; PE: Phycoerythrin; PI: Propidium iodide; PMA: Phorbol 12-myristate 13-acetate; RA: Rheumatoid arthritis; RER: Respiratory exchange ratio; RPMl: Roswell Park Memorial Institute; SBAS: Stanford Brief Activity Survey; TLR: Toll-like receptor; TNF: Tumor necrosis factor; TUG: Timed Up and Go; VAS: Visual analogue scale for pain;

$\mathrm{VO}_{2 \text { peak: }}$ Aerobic capacity

\section{Acknowledgements}

We acknowledge the participants and the staff members at the Duke Center for Living for their help with training and with recording of data. We appreciate the support of the Division of Rheumatology and Immunology at Duke University and, of course, all the participants.

\section{Funding}

This work was funded by an EU Marie Curie Outgoing Fellowship Grant (to DBB and JML) (PIOF-GA-2013-629981), a Duke University internal award (to DBB and $\mathrm{KMH}$ ) (NIH-UL1TR001117), and other internal research funds.

\section{Availability of data and materials}

The datasets generated and/or analyzed during the present study are not publicly available, owing to the risk of disclosure or deduction of private individual information, but they are available from the corresponding author on reasonable request.

\section{Authors' contributions}

$\mathrm{DBB}, J \mathrm{ML}$, WEK , and $\mathrm{KMH}$ conceived of and designed the study and experimental approach. DBB performed the immunological experiments. GS analyzed the results in a blinded fashion. DBB and JM conducted microscopy. DBB, LHW, CAS, and AH performed the physiological and functional testing and exercise training of participants. JLH and VBK completed the inflammatory cytokine analysis. MLM completed the plasma fatty acid analysis. LK screened and recruited all the participants. KMH assisted in statistical analyses. DBB wrote the manuscript. All authors contributed to the final manuscript. All authors read and approved the final manuscript.

Ethics approval and consent to participate

All participants gave written informed consent, and the study was approved by the Duke University Medical Center Institutional Review Board (IRB no. Pro00064057).

\section{Competing interests}

The sponsors had no role in study design, data collection and analysis, decision to publish, or preparation of the manuscript. All authors declare that they have no competing interests.

\section{Publisher's Note}

Springer Nature remains neutral with regard to jurisdictional claims in published maps and institutional affiliations.

\section{Author details}

${ }^{1}$ Duke Molecular Physiology Institute, Duke University School of Medicine, Durham, NC, USA. 'Division of Medical Oncology, Duke University School of Medicine, Durham, NC 27701, USA. ${ }^{3}$ MRC-ARUK Centre for Musculoskeletal Ageing Research, Institute of Inflammation and Ageing, University of Birmingham, Birmingham, UK. ${ }^{4}$ Department of Kinesiology, Louisiana State University, Baton Rouge, LA, USA. ${ }^{5}$ NIHR Birmingham Biomedical Research Centre in Inflammation, University Hospital Birmingham, Birmingham, UK

Received: 1 March 2018 Accepted: 14 May 2018

Published online: 14 June 2018

References

1. Mclnnes IB, Schett $G$. The pathogenesis of rheumatoid arthritis. N Engl J Med. 2011;365(23):2205-19.

2. Weyand CM, Goronzy JJ. Aging of the immune system: mechanisms and therapeutic targets. Ann Am Thorac Soc. 2016;13(Suppl 5):S422-8.

3. Mazzone A, et al. Immunomodulation of neutrophil chemotaxis in rheumatoid arthritis using levamisole and methisoprinol. Clin Ther. 1986;8(2):232-7. 


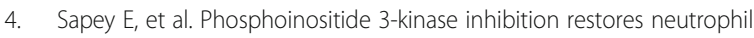
accuracy in the elderly: towards targeted treatments for immunosenescence. Blood. 2014;123(2):239-48.

5. Cedergren J, et al. Intracellular oxidative activation in synovial fluid neutrophils from patients with rheumatoid arthritis but not from other arthritis patients. J Rheumatol. 2007;34(11):2162-70.

6. Khandpur R, et al. NETs are a source of citrullinated autoantigens and stimulate inflammatory responses in rheumatoid arthritis. Sci Transl Med. 2013;5(178):178ra40

7. Fairhurst AM, et al. Rheumatoid peripheral blood phagocytes are primed for activation but have impaired Fc-mediated generation of reactive oxygen species. Arthritis Res Ther. 2007;9(2):R29.

8. Udalova IA, Mantovani A, Feldmann M. Macrophage heterogeneity in the context of rheumatoid arthritis. Nat Rev Rheumatol. 2016;12(8):472-85.

9. Shaw $A C$, et al. Aging of the innate immune system. Curr Opin Immunol. 2010;22(4):507-13.

10. Rossol M, et al. The CD14 ${ }^{\text {bright }} \mathrm{CD} 16+$ monocyte subset is expanded in rheumatoid arthritis and promotes expansion of the Th17 cell population. Arthritis Rheum. 2012;64(3):671-7.

11. Iwahashi M, et al. Expression of Toll-like receptor 2 on CD16+ blood monocytes and synovial tissue macrophages in rheumatoid arthritis. Arthritis Rheum. 2004;50(5):1457-67.

12. Gierut A, Perlman H, Pope RM. Innate immunity and rheumatoid arthritis. Rheum Dis Clin North Am. 2010;36(2):271-96.

13. Redlich K, Smolen JS. Inflammatory bone loss: pathogenesis and therapeutic intervention. Nat Rev Drug Discov. 2012;11(3):234-50.

14. Arleevskaya Ml, et al. Mononuclear phagocytes in rheumatoid arthritis patients and their relatives - family similarity. Open Rheumatol J. 2011:5:36-44.

15. Bongartz T, et al. Anti-TNF antibody therapy in rheumatoid arthritis and the risk of serious infections and malignancies: systematic review and meta-analysis of rare harmful effects in randomized controlled trials. JAMA 2006;295(19):2275-85.

16. Veldhuijzen van Zanten JJ, et al. Perceived barriers, facilitators and benefits for regular physical activity and exercise in patients with rheumatoid arthritis: a review of the literature. Sports Med. 2015:45(10):1401-12.

17. Swardh E, Brodin N. Effects of aerobic and muscle strengthening exercise in adults with rheumatoid arthritis: a narrative review summarising a chapter in physical activity in the prevention and treatment of disease (FYSS 2016). Br J Sports Med. 2016;50(6):362-7.

18. Gleeson $M$, et al. The anti-inflammatory effects of exercise: mechanisms and implications for the prevention and treatment of disease. Nat Rev Immunol. 2011;11(9):607-15.

19. Petersen AM, Pedersen BK. The anti-inflammatory effect of exercise. J Appl Physiol. 2005;98:1154-62

20. Bartlett DB, et al. Habitual physical activity is associated with the maintenance of neutrophil migratory dynamics in healthy older adults. Brain Behav Immun. 2016;56:12-20.

21. Slentz CA, et al. Effects of exercise training alone vs a combined exercise and nutritional lifestyle intervention on glucose homeostasis in prediabetic individuals: a randomised trial. Diabetologia. 2016:59(10):2088-98.

22. Bartlett $\mathrm{DB}$, et al. Association of the composite inflammatory biomarker GlycA, with exercise-induced changes in body habitus in men and women with prediabetes. Oxid Med Cell Longev. 2017;2017:5608287.

23. Kraus WE, et al. Effects of the amount and intensity of exercise on plasma lipoproteins. N Engl J Med. 2002;347(19):1483-92.

24. Stewart LK, et al. Influence of exercise training and age on CD14+ cell-surface expression of Toll-like receptor 2 and 4. Brain Behav Immun. 2005;19(5):389-97.

25. Bartlett DB, et al. Neutrophil and monocyte bactericidal responses to 10 weeks of low-volume high-intensity interval or moderate-intensity continuous training in sedentary adults. Oxid Med Cell Longev. 2017;2017:8148742.

26. McFarlin BK, et al. TLR4 is lower in resistance-trained older women and related to inflammatory cytokines. Med Sci Sports Exerc. 2004;36(11):1876-83.

27. Gibala MJ, et al. Physiological adaptations to low-volume, high-intensity interval training in health and disease. J Physiol. 2012:590(5):1077-84.

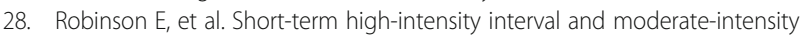
continuous training reduce leukocyte TLR4 in inactive adults at elevated risk of type 2 diabetes. J Appl Physiol (1985). 2015;119(5):508-16.
29. Durrer $C$, et al. Acute high-intensity interval exercise reduces human monocyte Toll-like receptor 2 expression in type 2 diabetes. Am J Physiol Regul Integr Comp Physiol. 2017;312(4):R529-38.

30. Sandstad J, et al. The effects of high intensity interval training in women with rheumatic disease: a pilot study. Eur J Appl Physiol. 2015;115(10):2081-9.

31. Baslund $B$, et al. Effect of 8 wk of bicycle training on the immune system of patients with rheumatoid arthritis. J Appl Physiol (1985). 1993;75(4):1691-5.

32. van den Ende $\mathrm{CH}$, et al. Comparison of high and low intensity training in well controlled rheumatoid arthritis: results of a randomised clinical trial. Ann Rheum Dis. 1996;55(11):798-805.

33. Arnett FC, et al. The American Rheumatism Association 1987 revised criteria for the classification of rheumatoid arthritis. Arthritis Rheum. 1988:31(3):315-24.

34. Howley ET. Type of activity: resistance, aerobic and leisure versus occupational physical activity. Med Sci Sports Exerc. 2001:33(6 Suppl):S364-9. discussion S419-20.

35. AbouAssi $\mathrm{H}$, et al. Adipose depots, not disease-related factors, account for skeletal muscle insulin sensitivity in established and treated rheumatoid arthritis. J Rheumatol. 2014:41(10):1974-9.

36. Siri WE. Body composition from fluid spaces and density: analysis of methods. 1961. Nutrition. 1993;9(5):480-91. discussion 480, 492

37. Dempster P, Aitkens S. A new air displacement method for the determination of human body composition. Med Sci Sports Exerc. 1995;27(12):1692-7.

38. Nordin E, et al. Prognostic validity of the Timed Up-and-Go test, a modified Get-Up-and-Go test, staff's global judgement and fall history in evaluating fall risk in residential care facilities. Age Ageing. 2008;37(4):442-8.

39. Muir SW, et al. Balance impairment as a risk factor for falls in communitydwelling older adults who are high functioning: a prospective study. Phys Ther. 2010;90(3):338-47.

40. Muir SW, et al. Use of the Berg Balance Scale for predicting multiple falls in community-dwelling elderly people: a prospective study. Phys Ther. 2008;88(4):449-59.

41. Diabetes Trials Unit, Oxford Centre for Diabetes, Endocrinology and Metabolism, University of Oxford. HOMA calculator. https://www.dtu.ox.ac. uk/homacalculator/. Accessed June 2014.

42. Wallace TM, Levy JC, Matthews DR. Use and abuse of HOMA modeling. Diabetes Care. 2004:27(6):1487-95.

43. Muinonen-Martin AJ, et al. An improved chamber for direct visualisation of chemotaxis. PLoS One. 2010;5(12):e15309.

44. Zhu $X$, et al. A surrogate method for assessment of $\beta_{2}$-integrin-dependent adhesion of human eosinophils to ICAM-1. J Immunol Methods. 2000;240(1-2):157-64.

45. Hazeldine J, et al. Impaired neutrophil extracellular trap formation: a nove defect in the innate immune system of aged individuals. Aging Cell. 2014;13(4):690-8.

46. Smith $\mathrm{BD}$, et al. Recommendations for the identification of chronic hepatitis $C$ virus infection among persons born during 1945-1965. MMWR Recomm Rep. 2012;61(RR-4):1-32.

47. Cooney JK, et al. Benefits of exercise in rheumatoid arthritis. J Aging Res. 2011;2011:681640.

48. Stenstrom $\mathrm{CH}$, Minor MA. Evidence for the benefit of aerobic and strengthening exercise in rheumatoid arthritis. Arthritis Rheum. 2003:49(3):428-34.

49. Plasqui $\mathrm{G}$. The role of physical activity in rheumatoid arthritis. Physiol Behav. 2008:94(2):270-5.

50. Fenton SAM, et al. Sedentary behaviour in rheumatoid arthritis: definition, measurement and implications for health. Rheumatology (Oxford). 2018;57(2):213-26.

51. Hakkinen A, et al. A randomized two-year study of the effects of dynamic strength training on muscle strength, disease activity, functional capacity, and bone mineral density in early rheumatoid arthritis. Arthritis Rheum. 2001:44(3):515-22

52. Hakkinen $A$, et al. Sustained maintenance of exercise induced muscle strength gains and normal bone mineral density in patients with early rheumatoid arthritis: a 5 year follow up. Ann Rheum Dis. 2004;63(8):910-6.

53. van den Ende $\mathrm{CH}$, et al. Effect of intensive exercise on patients with active rheumatoid arthritis: a randomised clinical trial. Ann Rheum Dis. 2000:59(8):615-21.

54. Komatireddy GR, et al. Efficacy of low load resistive muscle training in patients with rheumatoid arthritis functional class II and III. J Rheumatol. 1997;24(8):1531-9. 
55. Stavropoulos-Kalinoglou A, et al. Individualised aerobic and resistance exercise training improves cardiorespiratory fitness and reduces cardiovascular risk in patients with rheumatoid arthritis. Ann Rheum Dis. 2013;72(11):1819-25

56. Walsh NP, et al. Position statement part one: immune function and exercise. Exerc Immunol Rev. 2011;17:6-63.

57. Nieman DC, et al. Physical activity and immune function in elderly women. Med Sci Sports Exerc. 1993;25(7):823-31.

58. Rall LC, et al. Effects of progressive resistance training on immune response in aging and chronic inflammation. Med Sci Sports Exerc. 1996;28(11):1356-65.

59. den Broeder $\mathrm{AA}$, et al. Neutrophil migration and production of reactive oxygen species during treatment with a fully human anti-tumor necrosis factor-a monoclonal antibody in patients with rheumatoid arthritis. J Rheumatol. 2003;30(2):232-7.

60. Miesel R, Murphy MP, Kroger H. Enhanced mitochondrial radical production in patients which rheumatoid arthritis correlates with elevated levels of tumor necrosis factor a in plasma. Free Radic Res. 1996;25(2):161-9.

61. Talbot J, et al. CCR2 expression in neutrophils plays a critical role in their migration into the joints in rheumatoid arthritis. Arthritis Rheumatol. 2015;67(7):1751-9.

62. Fuente MDL, Hernanz A, Vallejo M. The immune system in the oxidative stress conditions of aging and hypertension: favorable effects of antioxidants and physical exercise. Antioxid Redox Signal. 2005;7(9-10):1356-66.

63. Syu GD, Chen HI, Jen CJ. Differential effects of acute and chronic exercise on human neutrophil functions. Med Sci Sports Exerc. 2012;44(6):1021-7.

64. Babior BM. Phagocytes and oxidative stress. Am J Med. 2000;109(1):33-44.

65. Hazeldine J, Lord J. Innate immunesenescence: underlying mechanisms and clinical relevance. Biogerontology. 2015;16(2):187-201.

66. de Siqueira MB, et al. Enhanced neutrophil phagocytic capacity in rheumatoid arthritis related to the autoantibodies rheumatoid factor and anti-cyclic citrullinated peptides. BMC Musculoskelet Disord. 2015;16:159.

67. Paino IM, et al. Phagocytosis and nitric oxide levels in rheumatic inflammatory states in elderly women. J Clin Lab Anal. 2011;25(1):47-51.

68. Turner RA, Schumacher R, Myers AR. Phagocytic function of polymorphonuclear leukocytes in rheumatic diseases. J Clin Invest. 1973;52(7):1632-5.

69. Wynne KM, et al. Cellular phagocytic studies in rheumatoid arthritis patients treated with levamisole. Ann Rheum Dis. 1981;40(4):382-7.

70. Okuda K, et al. An investigation of leukocyte function and phagocytosis of immune complexes in patients with rheumatoid arthritis. Jpn J Exp Med. 1975;45(1):1-10.

71. Wong KL, et al. Gene expression profiling reveals the defining features of the classical, intermediate, and nonclassical human monocyte subsets. Blood. 2011;118(5):e16-31.

72. Ziegler-Heitbrock $\mathrm{L}$, et al. Nomenclature of monocytes and dendritic cells in blood. Blood. 2010;116(16):e74-80.

73. Cros J, et al. Human CD14 ${ }^{\mathrm{dim}}$ monocytes patrol and sense nucleic acids and viruses via TLR7 and TLR8 receptors. Immunity. 2010;33(3):375-86.

74. Huang QQ, Pope RM. Role of Toll like receptors in rheumatoid arthritis. Curr Rheumatol Rep. 2009;11(5):357-64.

75. Bartlett $\mathrm{DB}$, et al. A novel inflammatory biomarker, GlycA, associates with disease activity in rheumatoid arthritis and cardio-metabolic risk in BMI-matched controls. Arthritis Res Ther. 2016;18(86)

76. Huffman $\mathrm{KM}$, et al. Response of high-sensitivity $\mathrm{C}$-reactive protein to exercise training in an at-risk population. Am Heart J. 2006;152(4):793-800.

77. Krüger $\mathrm{K}$, et al. Exercise-induced redistribution of $\mathrm{T}$ lymphocytes is regulated by adrenergic mechanisms. Brain Behav Immun. 2008;22(3):324-38.

78. Kruger K, Mooren FC. T cell homing and exercise. Exerc Immunol Rev. 2007;13:37-54

\section{Ready to submit your research? Choose BMC and benefit from:}

- fast, convenient online submission

- thorough peer review by experienced researchers in your field

- rapid publication on acceptance

- support for research data, including large and complex data types

- gold Open Access which fosters wider collaboration and increased citations

- maximum visibility for your research: over $100 \mathrm{M}$ website views per year

At BMC, research is always in progress.

Learn more biomedcentral.com/submissions 